\title{
Safety of transtympanic application of probiotics in a chinchilla animal model
}

\author{
Carol Nhan ${ }^{1}$, Aren Bezdjian², Shyamali Saha ${ }^{3}$, Satya Prakash³, Lily H. P. Nguyen ${ }^{4}$ and Sam J. Daniel ${ }^{1^{*}}$
}

\begin{abstract}
Background: Chronic suppurative otitis media can be recalcitrant and difficult to treat, particularly with the increasing occurrence of antibiotic resistance. Lactobacillus plantarum is a probiotic that has been shown to decrease S. aureus and $P$. aeruginosa growth in wounds, making it a good candidate for the treatment of chronic suppurative otitis media. However, before it can be applied in the ear, its ototoxicity potential must be evaluated.

Methods: A prospective controlled trial was conducted in a chinchilla animal model at the Animal care research facilities of the Montreal Children's Hospital Research Institute to determine whether Lactobacillus plantarum is ototoxic when applied transtympanically. Ten chinchillas each had one ear randomly assigned to receive $10^{9} \mathrm{CFU} / \mathrm{mL}$ of Lactobacillus plantarum solution, while the contralateral ear received saline. Auditory brainstem responses were measured bilaterally at 8,20,25 kHz before, at 7-10 days after application, and at 28 days after application of probiotic or saline. Facial nerve and vestibular function were assessed clinically.
\end{abstract}

Results: There were no statistically significant differences in hearing thresholds between control and experimental ears at 28 days after application. A difference of $11 \mathrm{~dB}$ was noted in the $25 \mathrm{kHz}$ range at day 7-10, but resolved by day 28 . No animals receiving probiotics developed vestibular nerve dysfunction. There was no histologic evidence of auditory hair cell damaged evidenced by scanning electron microscopy.

Conclusion: Our study suggests that a single application of Lactobacillus plantarum at $10^{9} \mathrm{CFU} / \mathrm{mL}$ does not cause ototoxicity in a chinchilla animal model. These preliminary safety evaluations and the pathogen inhibitory effects of $L$. plantarum demonstrated by previous studies present this probiotic as a candidate of interest for further investigation.

Keywords: Probiotic, Otitis, Ototoxicity, Transtympanic, Chinchilla

\section{Background}

Chronic suppurative otitis media (CSOM) can be challenging to treat, particularly when complicated by antibiotic resistance or secondary otomycosis. It is the leading cause of childhood hearing impairment in the developing countries [1] and has had serious implications for speech and language development in children [1-3], impacting cognitive and education outcomes [4]. Serious complications arising from these infections can cause meningitis, intracranial abscess, facial palsy, and lateral sinus thrombosis [5].

The majority of infections are polymicrobial and involve chronic inflammation of the middle ear. Pathogens

\footnotetext{
* Correspondence: sam.daniel@mcgill.ca

'Department of Otolaryngology - Head \& Neck Surgery and McGill Auditory Sciences Laboratory, The Montréal Children's Hospital, McGill University, 1001 Boulevard Décarie, Montréal, Québec H4A 3J1, Canada

Full list of author information is available at the end of the article
}

most commonly associated with CSOM are Pseudomonas, Staphylococcus, Peptostreptococcus, Fusobacterium, Prevotella, and Porphyromonas [6].

Currently, the first-line treatment for uncomplicated CSOM involves antibiotics and anti-inflammatory agents applied topically to the ear. This treatment best achieves the highest dose delivery with the least secondary effects [7]. However, overuse of antibiotics has resulted in resistant pathogens. Moreover, prolonged use of antibiotics has been associated with development of otomycosis [8].

Probiotics are living microorganisms that can provide beneficial effects [9]. For over a decade, probiotic bacteria have successfully treated infections typically related to gastro-intestinal (GIT) diseases [10]. More recently, several non-GIT applications have been investigated [11]. In children with recurrent otitis media, nasal spray applications of probiotics have been shown to reduce 
their rates of both infection and middle ear effusion [12]. It is hypothesized that probiotics help restore the native polymicrobial population in the nasopharynx, which typically shows reduced levels of nasopharyngeal commensals in cases of recurrent acute otitis media [13]. These studies bring into question whether topical probiotic bacteria application could also be beneficial in the prevention and treatment of CSOM.

Lactobacillus plantarum is a probiotic shown to prevent both $S$. aureus and $P$. aeruginosa, the main organisms found in CSOM [6], from establishing wound infections [14]. It also has antagonistic activity against Peptostreptococcus [15], an anaerobic organism commonly found in COSM [6]. In a comparison of three strains of lactobacillus, Lactobacillus plantarum was found to have the best inhibitory activity against $S$. aureus and $P$. aeruginosa [14] making it a good potential candidate for the treatment of CSOM. Nonetheless, the ototoxicity potential of probiotics remains to be established prior to investigating otic applications. Thus, before studying whether Lactobacillus plantarum is effective in treating CSOM, its safety when applied topically to the middle ear must be determined.

\section{Methods}

\section{Study overview}

Chinchilla were used to verify the ototoxity of L. plantarum. The chinchilla was chosen because it is a wellestablished animal model for hearing loss studies [16-18]. They also have large tympanic membranes and middle ears similar to humans and a cochlea that is readily dissectible. Each chinchilla had a single application of a solution of probiotic transtympanically to the randomly selected experimental ear and phosphate buffered saline (PBS) to the control ear. Hearing was assessed by auditory brainstem responses (ABR) prior to experimental application of probiotic, then at early and late intervals following application. The animals were euthanized and the cochlear structures were analyzed using scanning electron microscopy (SEM).

\section{Animal care and ethics}

The study received approval by the Animal Care Committee of the McGill University Health Centre Research Institute and was conducted at the McGill Auditory Sciences Laboratory in accordance with the guidelines of the Canadian Council for Animal Care. Ten female chinchillas (C. laniger, Ryerson Chinchilla Ranch, $\mathrm{OH}$ ) were included in the study. Throughout the study, chinchillas were kept in temperature and light controlled rooms with free access to water and commercial food by the animal care research facilities of the Montreal Children's Hospital Research Institute.

\section{Sample size}

The sample size of seven was calculated setting power at $80 \%$ and an alpha of 0.05 to show a difference of $20 \mathrm{~dB}$ with a standard deviation of $12.6 \mathrm{~dB}$ determined on a pilot study. Ten animals were used to account for the potential of animal loss during the study.

\section{Hearing evaluation}

Hearing evaluations of the chinchilla were performed at three different times: at baseline prior to application of the probiotic bacteria, early (day 7-10) and late (day 28) after application of probiotic. Hearing was tested by ABR on chinchilla anesthetized by $5 \%$ Isoflurane and maintained with $3 \%$ Isoflurane. Acoustic stimuli of 8000 , 20,000 , and $25,000 \mathrm{~Hz}$ pure tone bursts were presented to the chinchilla through insert earphones starting at $80 \mathrm{~dB}$ intensity and decreasing by $5 \mathrm{~dB}$ until a threshold was reached.

\section{Probiotic bacteria preparation}

L. plantarum ATCC 10241 was plated using MRS agar from an $80 \%(\mathrm{v} / \mathrm{v})$ frozen MRS-glycerol stock. The plate was incubated for $24 \mathrm{~h}$ at $37{ }^{\circ} \mathrm{C}$ with $5 \% \mathrm{CO}_{2}$ to ensure purity. A single colony from the MRS-agar plate was incubated for $24 \mathrm{~h}$ at $37^{\circ} \mathrm{C}$ in $10 \mathrm{~mL}$ of MRS broth. A standard curve was derived using the overnight culture of the bacteria to make a solution of $10^{9}$ colony-forming-units $(\mathrm{CFU}) / \mathrm{mL}$ in PBS for transtympanic application. The CFU count of the solution administered was determined again by standard colony counting to ensure accurate dosing. The $\mathrm{pH}$ and electrolyte content of the administered solution was verified in order to ensure no confounders in the ototoxicity study.

\section{Transtympanic application}

Each of 10 animals had one ear randomized to receive a single application of probiotic bacteria (experimental), while the contralateral ear received a single application of PBS (control). After anesthetising, a radial incision in the antero-inferior quadrant of the tympanic membrane was made and $0.4-0.7 \mathrm{~mL}$ of probiotic solution (until the middle ear was filled) was administered into the middle ear via a soft sterile polyethylene tubing catheter. The same volume of PBS was instilled into the control ears following the same protocol.

\section{Middle ear examination and histology}

Four weeks after application of the probiotic, all animals were euthanized. The middle ears were examined for bony or mucosal changes. The cochleae were dissected and fixed in $4 \%$ paraformaldehyde. Post-fixation staining with osmium tetroxide and graded dehydration with 30, 50, 70, 80,90 , and $100 \%$ alcohol was performed. Specimens were critical-point dried using Leica CPD 030, mounted, gold 
plated, and viewed using the Hitachi field emission electron microscopy (Hitachi S4700, Tokyo, Japan).

\section{Statistical analysis}

Early (day 7-10) and late (day 28) shifts in ABR thresholds after application of the probiotics were compared using paired T-test between the experimental and control ears across all three frequencies tested $(8,20,25 \mathrm{kHz})$. A $p$ value $<0.05$ was considered statistically significant.

\section{Results}

\section{Probiotic preparation and dose selection}

Previous studies testing the activity of Lactobacillus plantarum against Staphyloccus aureus or Pseudomas aeruginosa on wounds used concentrations of $10^{5}$ to $1.5 \times 10^{8}$ CFU/ml [19-21]. Standard colony counting of an aliquot of the probiotic solution used gave a count of $1.5 \times 10^{9}$ $\mathrm{CFU} / \mathrm{mL}$. The solution had a neutral $\mathrm{pH}$ of 7.0. $\mathrm{Na}+$ was $156 \mathrm{mmol} / \mathrm{L}, \quad \mathrm{K}+1.7 \mathrm{mmol} / \mathrm{L}$, and $\mathrm{Cl}$ $148 \mathrm{mmol} / \mathrm{L}$.

\section{Observations for physical signs of toxicity}

Three animals had to be euthanized before completion of the experiment due to unrelated illness and were therefore excluded from analysis. The remaining seven animals were in good health until the end of the experiment, maintaining steady weight gain and normal behaviors. Commonly accepted physical signs of ototoxicity are evidence of damage to cochleovestibular nerve, resulting signs of vestibular disturbance such as head tilt or disequilibrium.

\section{Auditory brainstem response threshold shifts}

To investigate ototoxicity, baseline hearing measured prior to application of solutions were compared to early post-application (day 7-10) and late post-application (day 28) using ABRs. On the early assessment (day 7-10 following transtympanic application of solution) a significant threshold shift was found at $25 \mathrm{kHz}$ in the ear with the test probiotic doses $(9.6 \pm 2.3 \mathrm{~dB})$ when compared to the control ear receiving $(-1.4 \pm 3.5 \mathrm{~dB}), p=$ 0.02 . This threshold shift resolved by the day 28 ABR measurements. No significant long-term hearing loss was observed between experimental and control ears at all frequencies and time points tested (Fig. 1). ABR data per animal is shown in Additional file 1: Table S1.

\section{Assessment of structural anatomy}

Day 1 following application of solutions transtympanically, examination of the tympanic membranes under anesthesia confirmed that the middle ears were still fluid-filled. Prior to measuring early ABRs at day 7-10, ears were again examined otoscopically revealing small amounts of effusions remaining. After euthanasia and temporal bone dissection examination revealed no mucosal changes in the bulla of experimental and control ears.

\section{Histology}

Three randomly selected pairs of cochlea were examined under SEM, which revealed no observable changes to the cochlear hair cells between the experimental and control ears for each animal. The three rows of outer hair cells in the Organ of Corti were intact in both the control and experimental ears (Fig. 2).

\section{Discussion}

Treatment of CSOM is problematic particularly when there is antibiotic resistance. Based on studies showing that probiotics can treat various infectious diseases [10-12], the question of whether probiotics could be effective in the treatment of CSOM is raised. Recolonization of the nasopharynx with commensal bacteria has been suggested as a strategy to treat recurrent otitis media [10, 11, 22]. Probiotic bacteria may be a safe and effective adjunct treatment for CSOM.

To date, topical application of probiotic directly to the ear has not been explored. An ideal probiotic would be effective against the pathogens common to CSOM, and have low potential of pathogenicity and ototoxicity. Studies show that $L$. plantarum is active against $P$. aeruginosa and $S$. aureus on wounds $[14,20,21]$ and active against methicillin-resistant $S$. aureus in vitro [19], making it a good candidate for treating recalcitrant CSOM. However, the safety of its use in the ear precludes study of its efficacy particularly since some bacteria, such as $S$. pneumoniae and $H$. influenzae, are known to have the potential to cause sensorineural hearing loss through virulence factors [23], bacterial toxins [24], or inflammatory mediators [25]. This explains the association of otitis media with sensorineural hearing loss [26-28].

This study is the first to demonstrate that the probiotic $L$. plantarum at a concentration of $1.5 \times 10^{9} \mathrm{CFU} / \mathrm{mL}$ applied a single time to the middle ear is not ototoxic as evidenced by $A B R$ results 28 days post-application and by electron microscopy of the cochlea. Such a study is particularly relevant for an organism that is not pro-inflammatory, such as L. plantarum, since a non-inflammatory state tends to allow easier permeability through the round window membrane and greater risk of ototoxicity. Since $L$. plantarum has demonstrated ability to limit growth of $P$. aeruginosa, $S$. aureus [20] and Peptrostreptococcus [21], it may be a candidate for further studies investigating its safety and therapeutic use in recalcitrant CSOM.

Inhibition of $P$. aeruginosa growth, as well as inhibition of the production of biofilm and elastase by a $10^{5} \mathrm{CFU} / \mathrm{mL}$ solution L. plantarum has been demonstrated both in vitro and in vivo [20]. In a burned-mouse model where burn wounds were infected with $P$. aeruginosa, $10^{6} \mathrm{CFU} / \mathrm{mL} L$. 


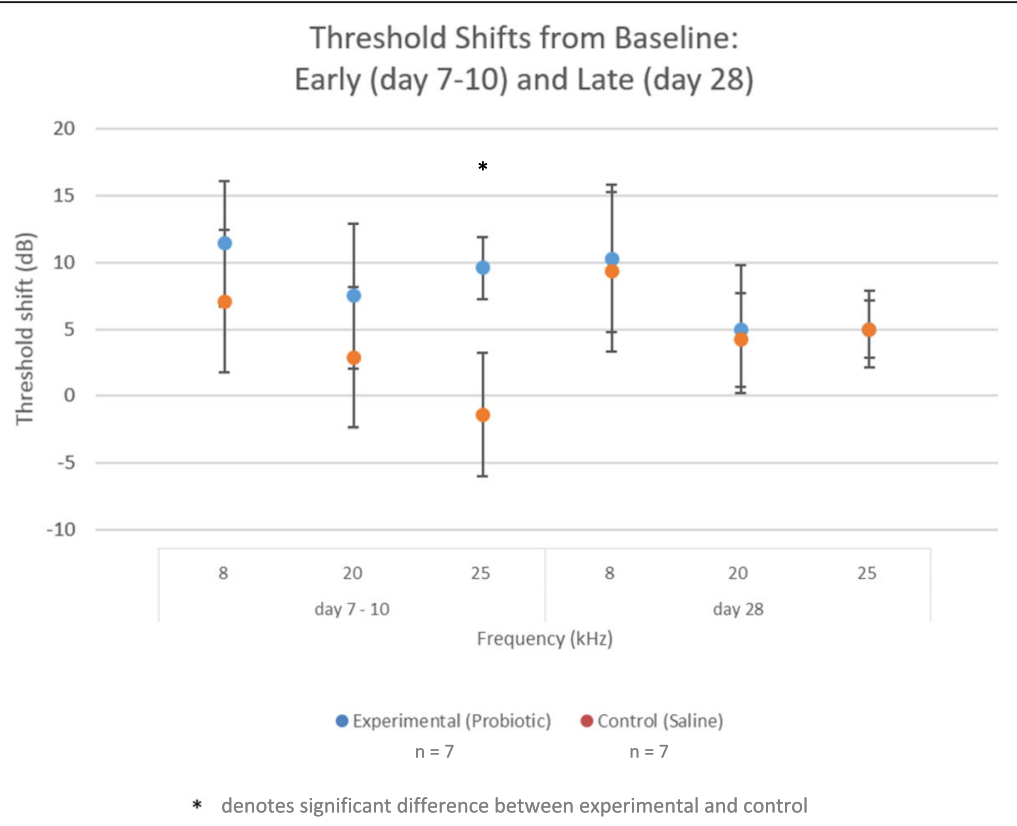

Fig. 1 Auditory brainstem response thresholds shifts from baseline. Error bars = 1 standard deviation. Abbreviations: dB, decibels; exp., experimental (probiotic); ctl, control (phosphate buffered saline)

plantarum applied topically to wounds led to decreased pathogen growth and improved healing. A study of burn patients suggested that topical $L$. plantarum at a concentration of $10^{5} \mathrm{CFU} / \mathrm{mL}$ was as effective as silver sulfadiazine (the gold standard in topical burn treatment) in decreasing bacterial load and promotion of wound healing [21].

A $1.5 \times 10^{9} \mathrm{CFU} / \mathrm{mL}$ solution of $L$. plantarum, a dose greater than that used in the studies on burn wounds, was administered once into the middle ear cavity and confirmed not to cause any toxicity to Chinchilla cochlear structures. The transient statistically significant difference in threshold shift between experimental and control ears seen at $25 \mathrm{kHz}$ during the early (day 7-10) ABR was likely due to the greater viscosity of the probiotic solution compared to PBS. It was noted on physical examination that the experimental ear demonstrated residual effusion at the time of early ABR. Even then this threshold shift was not considered clinically significant at only $11 \mathrm{~dB}$, and most importantly was resolved by day 28 .
Although this study serves as preliminary evidence that $L$. plantarum is safe for a single application directly to the middle ear, more complete testing must be done to confirm its safety and effectiveness in humans. Currently L. plantarum is widely used in fermented food products including yogurts, as well as probiotic supplements.

Limitations of this study include its small sample size, restricting its ability to detect hearing losses less than $20 \mathrm{~dB}$, and the single application of probiotic rather than multiple applications at intervals, making its findings preliminary in nature. Further investigations evaluating L. plantarum's otologic safety and clinical efficacy are needed. It would also be prudent to study its ototoxicity when used in an animal model of CSOM since that may alter release of cytokines and other factors which may impact ototoxicity. On the other hand, chronic otitis media causes an increase in the thickness of the round window membrane by a factor of two, which could have a protective effect due to decreased permeability of the round window membrane [29-31].

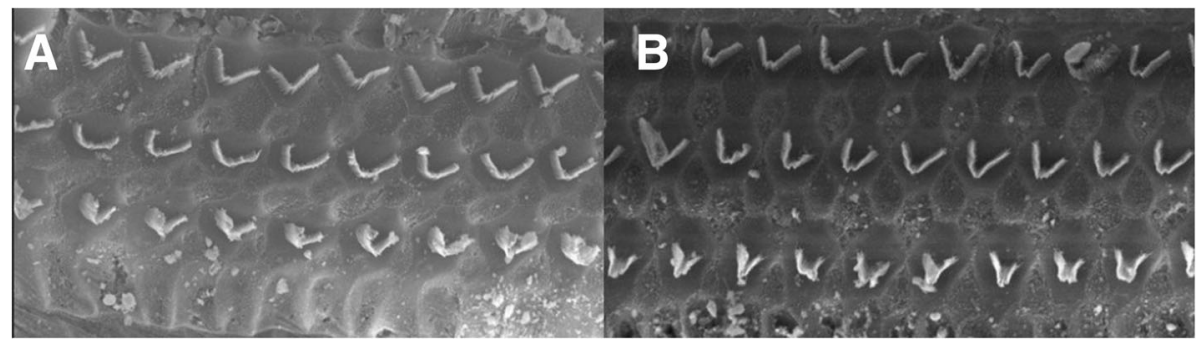

Fig. 2 Scanning electron microscopy showing comparison between control (a) and experimental (b) histological micrographs 


\section{Conclusion}

This study demonstrates that a $1.5 \times 10^{9} \mathrm{CFU} / \mathrm{mL}$ solution of L. plantarum applied a single time intratympanically is not ototoxic in a chinchilla animal model. Based on in vitro, animal, and burn wound studies, this probiotic bacteria could potentially be effective candidate in treating CSOM caused by $P$. aeruginosa and $S$. aureus. Hence, this study's preliminary otologic safety evaluations and the pathogen inhibitory effects of L. plantarum demonstrated by other groups present this probiotic as a candidate for further investigation in treating recalcitrant CSOM. Further pre-clinical and clinical investigations to understand the mechanism of actions responsible for such effects, its safety, and efficacy studies will be invaluable for determining whether L. plantarum could be used therapeutically in COSM.

\section{Additional file}

Additional file 1: Table S1. Auditory brainstem response threshold shifts $(d B)$ in the control (phosphate buffered saline) and experimental (probiotic) ears at 7-10 (Early) and 28 (Late) days post application of probiotic. (DOCX $16 \mathrm{~kb}$ )

\section{Abbreviations}

${ }^{\circ} \mathrm{C}$ : Degrees celcius; ABR: Auditory brainstem responses; CFU: Colony forming unit; CSOM: Chronic suppurative otitis media; dB: Decibel; Hz: Hertz; kHz: Kilohertz; L: Litre; mL: Millilitre; P. aeruginosa: Pseudomas aeruginosa; PBS: Phosphate buffered saline; S. aureus: Staphyloccus aureus; SEM: Scanning electron microscopy; v/v: Volume/volume

\section{Acknowledgments}

Not applicable.

\section{Funding}

The study was supported by the McGill University Head \& Neck Fund and the Fonds de Recherche Santé Québec.

\section{Availability of data and materials}

Data analysed during this study are included in this published article. Additional gathered data generated during the current study are available from the corresponding author on reasonable request.

\section{Authors' contributions \\ CN contributed in the study design, gathering and analysis of data and drafted the manuscript. $A B$ contributed in the study design, gathering and analysis of date. SS contributed in the preparation and maintenance of probiotic and expertise in probiotic research. SP contributed in the preparation and maintenance of probiotic and expertise in probiotic research. LN contributed in the study design. SD contributed in the conception of the study and analysis of the gathered data. All authors read and approved the final manuscript and are accountable for the work.}

\section{Ethics approval}

The study received approval by the Animal Care Committee of the McGil University Health Centre Research Institute. The McGill Auditory Sciences Laboratory conducts their in vivo animal studies in accordance with the guidelines of the Canadian Council for Animal Care.

\section{Consent for publication}

Not applicable.

\section{Competing interests}

The authors declare that they have no competing interests.

\section{Publisher's Note}

Springer Nature remains neutral with regard to jurisdictional claims in published maps and institutional affiliations.

\section{Author details}

${ }^{1}$ Department of Otolaryngology - Head \& Neck Surgery and McGill Auditory Sciences Laboratory, The Montréal Children's Hospital, McGill University, 1001 Boulevard Décarie, Montréal, Québec H4A 3J1, Canada. ²Department of Experimental Surgery and McGill Auditory Sciences Laboratory, The Montréal Children's Hospital, McGill University, Montréal, Québec, Canada.

${ }^{3}$ Departments of Biomedical Engineering, Physiology, and Artificial Cells and Organs Research Center, Biomedical Technology and Cell Therapy Research Laboratory, McGill University, Montréal, Québec, Canada. ${ }^{4}$ Department of Otolaryngology - Head \& Neck Surgery and Center for Medical Education, McGill University, Montreal, Québec, Canada.

Received: 10 April 2017 Accepted: 8 November 2017

Published online: 22 November 2017

\section{References}

1. Madana J, Yolmo D, Kalaiarasi R, Gopalakrishnan S, Sujatha S. Microbiological profile with antibiotic sensitivity pattern of cholesteatomatous chronic suppurative otitis media among children. Int J Pediatr Otorhinolaryngol. 2011;75(9):1104-8.

2. Daly KA, Hunter LL, Giebink GS. Chronic otitis media with effusion. Pediatrics in review. Am Acad Pediatr. 1999;20(3):85-93.

3. Bowd AD. Otitis media: health and social consequences for aboriginal youth in Canada's north. Int J Circumpolar Health. 2005;64(1):5-15.

4. Williams CJ, Jacobs AM. The impact of otitis media on cognitive and educational outcomes. Med J Aust. 2009;191(9 Suppl):S69-72.

5. Dubey SP, Larawin V. Complications of chronic suppurative otitis media and their management. Laryngoscope. 2007;117(2):264-7.

6. Brook J. The role of anaerobic bacteria in chronic suppurative otitis media in children: implications for medical therapy. Anaerobe. 2008;14(6):297-300.

7. Daniel S. Topical treatment of chronic Suppurative Otitis media. Curr Infect Dis Rep. 2012;14:121-7.

8. Jackman A, Ward R, April M, Bent J. Topical antibiotic induced otomycosis Int J Pediatr Otorhinolaryngol. 2005;69(6):857-60.

9. Sanders ME. Probiotics: definition, sources, selection, and uses. Clin Infect Dis. 2008;46(Suppl 2):S58-61. discussion S144-S151

10. Jones ML, Martoni CJ, Ganopolsky JG, Sulemankhil I, Ghali P, Prakash S. Improvement of gastrointestinal health status in subjects consuming lactobacillus reuteri NCIMB 30242 capsules: a post-hoc analysis of a randomized controlled trial. Expert Opin Biol Ther. 2013;13(12):1643-51.

11. Bernstein JM, Sagahtaheri-Altaie S, Dryja DM, Wactawski- Wende J. Bacterial interference in nasopharyngeal bacterial flora of otitis-prone and non-otitisprone children. Acta Otorhinolaryngol Belg. 1994;48(1):1-9.

12. Skovbjerg S, Roos K, Holm SE, Grahn Håkansson E, Nowrouzian F, Ivarsson M, Adlerberth I, Wold AE. Spray bacteriotherapy decreases middle ear fluid in children with secretory otitis media. Arch Dis Child. 2009;94(2):92-8

13. Berstein JM, Faden HF, Dryja DM, Wactawski-Wende J. Micro-ecology of the nasopharyngeal bacterial Flora in Otitis-prone and non-otitis-prone children. Acta Otolaryngol. 1993;13:88-92.

14. Al-Mathkury HJF, Al-Aubeidi HJR. Probiotic effect of lactobacilli on mice in wound infections. J Al-Nahrain Univ. 2008:11:111-6.

15. Strus M, Malinowska M. The range of antagonistic effects of lactobacillus bacterial strains on etiologic agents of bacterial vaginosis. Med Dosw Mikrobiol. 1999;51(1-2):47-57.

16. Nhan C, Bezdjian A, Alarfaj A, Daniel SJ. Can floseal ${ }^{\mathrm{TM}}$ be applied safely during otologic surgery? Assessment of ototoxicity in a chinchilla animal model. J Otolaryngol Head Neck Surg. 2017;29;46(1):24.

17. Nader ME, Kourelis M, Daniel SJ. Hydrogen peroxide ototoxicity in unblocking ventilation tubes: a chinchilla pilot study. Otolaryngol Head Neck Surg. 2007;136(2):216-20.

18. Aron M, Victoria Akinpelu O, Dorion D, Daniel S. Otologic safety of manuka honey. J Otolaryngol Head Neck Surg. 2012:41(Suppl 1):S21-30.17.

19. Brachkova MI, Marques P, Rocha J, Sepodes B, Duarte MA, Pinto JF. Alginate films containing lactobacillus plantarum as wound dressing for prevention of burn infection. J Hosp Infect. 2011;79(4):375-57. 
20. Valdez JC, Peral M, Rachid M, Santana M, Perdigon G. Interference of lactobacillus plantarum on Pseudomonas Aeruginosa in vitro and in infected burns. The potential use of probiotic in the wound treatment. ClinMicrobiol Infect. 2005;11:472-9.

21. Peral MC, Martinez MA, Valdez JC. Bacteriotherapy with lactobacillus plantarum in burns. Int Wound J. 2009;6(1):73-81.

22. Roos K, Håkansson EG, Holm S. Effect of recolonisation with "interfering" alpha streptococci on recurrences of acute and secretory otitis media in children: randomised placebo controlled trial. Brit Med J. 2001;322(7280):210-2.

23. Tsuprun V, Cureoglu S, Schachern PA, Ferrieri P, Briles DE, Paparella MM, Juhn SK. Role of pneumococcal proteins in sensorineural hearing loss due to otitis media. Otol Neurotol. 2008;29(8):1056-60.

24. Demaria TF. Localization of nontypeable Haemophilus influenza endotoxin in the middle and inner ear during experimental otitis media. Acta Otolaryngol. 1999:119(5):583-7.

25. Juhn SK, Jung TTK, Lin J, Rhee CK. Effect of inflammatory mediators on middle ear pathology and inner ear function. Ann N Y Acad Sci. 1997;830:130-42.

26. Margolis RH, Hunter LL, Saupe JR, Giebink GS. Effect of otitis media on extended high frequency hearing in children. Ann Otol Rhinol Laryngol. 1993;102:1-5

27. Papp Z, Rezes S, Sziklai I. Sensorineural hearing loss in chronic otitis media. Otol Neurotol. 2003:24:141-4

28. Morizono T, Giebink GS, Paparella MM, Sikora MA, Shea D. Sensorineural hearing loss in experimental purulent otitis media due to Streptococcus Pneumoniae. Arch Otolaryngol. 1985;111:794-8.

29. Sahni RS, Paparella MM, Schachern PA, Goycoolea MV, Le CT. Thickness of the human round window membrane in different forms of otitis media. Arch Otolaryngol Head Neck Surg. 1987;113:630-4.

30. Schachern PA, Paparella MM, Goycoolea MV, Duvall AJ III, Choo YB. The permeability of the round window membrane during otitis media. Arch Otolaryngol Head Neck Surg. 1987;113:625-9.

31. Schachern PA, Paparella MM, Goycoolea MV. Experimental grafting of the round window membrane: part I. Laryngoscope. 1987;97:790-6.

Submit your next manuscript to BioMed Central and we will help you at every step:

- We accept pre-submission inquiries

- Our selector tool helps you to find the most relevant journal

- We provide round the clock customer support

- Convenient online submission

- Thorough peer review

- Inclusion in PubMed and all major indexing services

- Maximum visibility for your research

Submit your manuscript at www.biomedcentral.com/submit
) Biomed Central 